\title{
Conflict of interest: what is it, and how do journals manage it in the publication process?
}

\author{
Steven Swift ${ }^{1}$ - Diaa Rizk ${ }^{2} \cdot$ Gunnar Lose $^{3}$
}

Received: 30 March 2017 / Accepted: 3 May 2017 /Published online: 25 May 2017

(C) The International Urogynecological Association 2017

Conflict of interest (COI) according to the World Association of Medical Editors (WAME) " ... exists when there is a divergence between an individual's private interests (competing interests) and his or her responsibilities to scientific and publishing activities such that a reasonable observer might wonder if the individual's behavior or judgment was motivated by considerations of his or her competing interests" [1]. Most individuals think of COI in financial terms, but in fact, it has as much to do with an individual's loyalty to a research concept, society, or clinical belief system as it does with any financial considerations. The WAME suggests that each journal have its own definition of COI and attempt to maintain a consistent policy in its publication process.

This editorial suggests that the International Urogynecology Journal (IUJ) begin adopting a formal process for reporting and managing COI among the editors, editorial board, reviewers, and authors. The intent is to guarantee the most transparent practice in scientific publication and provide the best and most balanced reviews for authors submitting manuscripts. COI does not necessarily disqualify a manuscript from review and ultimate publication as long as the COI is reported in a way that readers and policymakers can properly weigh its findings in

Steven Swift

swifts@musc.edu

1 Department of Obstetrics and Gynecology, Medical University of South Carolina, Charleston, SC, USA

2 Department of Obstetrics and Gynecology, College of Medicine and Medical Sciences, Arabian Gulf University, Manama, Bahrain

3 Herlev Hospital, Department of Obstetrics and Gynaecology, Herlev, and Institute for Clinical Medicine, University of Copenhagen, Copenhagen, Denmark light of other literature and if it can be managed with disclosure. We suggest adopting such policies to provide transparency, not to implement any form of censorship.

The following areas are consistent with the WAME policy statement on COI, and we believe they should be the backbone of IUJ's policy. In addition, the editors will adopt the form provided by the International Committee of Medical Journal Editors for declaring COIs [2].

Financial COI is present if an individual in the publication process has any expectation of receiving money, gifts, or services that would be related to a specific outcome in the publication process. For authors, this means accurately completing the COI form that accompanies any submission. For reviewers, this means either declaring a conflict of opinion and declining the review, or declaring the COI to the editor managing the manuscript.

Academic COI is present if an individual in the publication process has a strong belief (academic passion) in an explanation of a phenomenon, method, or idea. This COI is probably the most difficult to manage. For authors it means not overstating research outcomes. For reviewers, it means objectively reviewing an article even contrary to personal opinions and beliefs.

Personal COI is present if an individual in the publication process has a personal relationship (either positive or negative) such that it will unduly influence the outcome of the publication process. For reviewers, this means being honest and not allowing personal relationships to influence opinions and reviews.

Institutional COI is present when an individual in the publication process has a tie to an institution that may have a position or interest in the outcomes of a publication. This COI is similar to the financial COI and is particularly pertinent for authors employed by the corporation producing the report.

These areas of COI will become part of the IUJ's instructions to authors, and editors will recommend that 
manuscript reviewers refer to these recommendations if there are any questions regarding their participation in the review process.

Managing COIs with a process that is nonputative, fair, and leads to transparency is the goal of any process for declaring COI. If a COI is declared appropriately, it is often all that is necessary for managing the situation. However, there are rare instances in which a COI is not manageable. In these rare cases, the editors may disqualify the report from publication. If there are concerns that a COI was not properly declared, the editors will perform an investigation of the concern as outlined by WAME. If it is felt a COI was not properly declared, the editors will write the authors requesting a statement providing the reason, and that statement will will be published as an erratum to the article.

\section{References}

1. WAME Editorial Policy and Publication Ethics Committee. Journals' Role in Managing Conflict of Interest Related to the Funding of Research" and the WAME Publication Ethics Policy "Conflicts of Interest." July 2009. http://www.wame.org/about/ conflict-of-interest-in-peer-reviewed-medical.

2. Recommendations for the conduct, reporting, editing and publication of scholarly work in medical journals. ICJME Updated Report, December 2015. http://icmje.org/conflicts-of-interest/. 